\title{
Transnasal sphenopalatine ganglion block for post herpetic ophthalmic neuralgia: a case report
}

\begin{abstract}
Post herpetic ophthalmic neuralgia can often be a frustrating condition for both patients and physicians due to its medical treatment resistance. In this case report, we demonstrate the effectiveness of the transnasal sphenopalatine ganglion block in treating severe, debilitating post herpetic ophthalmic neuralgia. We utilized the transnasal technique with a cotton tipped applicator soaked in $4 \%$ lidocaine. This technique is advantageous in that it is noninvasive, well tolerated, technically simple, and easy to repeat if necessary. We propose an increased consideration of the transnasal sphenopalatine ganglion block for the treatment of post herpetic ophthalmic neuralgia.
\end{abstract}

Keywords: sphenopalatine ganglion block, sphenopalatine ganglion, pterygopalatine ganglion block, pterygopalatine ganglion, post herpetic ophthalmic neuralgia, post herpetic neuralgia
Volume 8 Issue 5 - 2017

\author{
Lance Brandenburgh, I Thomas Pittelkow2 \\ 'Department of Anesthesiology and Perioperative Medicine, \\ Mayo Clinic, USA \\ ${ }^{2}$ Department of Anesthesiology and Perioperative Medicine, \\ Division of Pain Medicine, Mayo Clinic, USA
}

\begin{abstract}
Correspondence: Lance Brandenburgh, MD, Department of Anesthesiology and Perioperative Medicine, Mayo Clinic, Rochester, MN, United States of America,

Email Brandenburgh.Lance@mayo.edu
\end{abstract}

Received: September 20, 2017| Published: September 29 2017

\section{Abbreviation: SPG, sphenopalatine ganglion}

\section{Introduction}

Post herpetic ophthalmic neuralgia can be a devastating diagnosis for patients, given the concern for compromised vision, profound disability, and refractory nature of it symptoms. Unfortunately, there is no definitive treatment and the primary focus is on symptom control. Typically, multiple different oral medication classes are initiated with the hope to reduce the neuropathic pain. Topical therapies are another treatment option, but this is often limited due to the sensitive nature of the tissues involved. Herein, we discuss the utility and application of a noninvasive and technically simple application of a transnasal sphenopalatine ganglion block to offer temporary and sustained relief of this facial pain syndrome.

\section{Case presentation}

A 57 year old female patient with a past medical history significant for mantle cell lymphoma, three months status post autologous peripheral blood stem cell transplantation, was transferred to our facility for severe right orbital pain in the setting of post herpetic ophthalmic neuralgia. She had previously been treated with two weeks of IV acyclovir. Over a five week period she was also treated with valganciclovir, gabapentin, duloxetine, prednisone, over the counter non-steroidal anti-inflammatory drugs, acetaminophen, and opioid analgesics. She unfortunately saw no improvement in her symptoms. A majority of her pain was located in the V1/V2 distribution, specifically at her right eye. Her pain was described as sharp, stabbing, tingling and constant with intermittent, frequent episodes of unbearable, lancinating pain. Her pain was also associated with tearing, photophobia, and intense pruritus over her right hemicranium in the V1 distribution. She was bedbound due to the severity of the discomfort. On admission, a cadre of medications were initiated, including Fentanyl PCA, acetaminophen, valganciclovir, prednisone, duloxetine, specialty compounded amitriptyline-ketamine-lidocaine cream, lubricating eye drops, and antihistamines, again with minimal relief in symptoms. On hospital day number three, the pain service was consulted. We performed a transnasal sphenopalatine ganglion block using $4 \%$ lidocaine soaked on a cotton tip applicator. She noted a considerable improvement in her symptoms immediately post block with sustained relief of over $50 \%$ reported during the subsequent days. She did endorse the presence of pain, photophobia, and pruritus although this was much less in both frequency and intensity. Her appetite and insomnia also improved and she was able to be discharged from the hospital three days post block.

\section{Discussion}

Post herpetic ophthalmic neuralgia can be a severe and debilitating condition. The diagnosis is clinical, and is described as a chronic, severe pain in a dermatomal distribution that continues after healing of a herpes zoster rash. Antibody testing can be considered if a rash is not evident prior to the characteristic symptoms. The pathophysiology likely involves reactivation of latent varicella-zoster virus in the trigeminal nerve with subsequent nerve damage. Symptoms may be intermittent or constant and may be described as aching, burning, pruritic, lancinating, or sharp. Symptoms may be associated with altered sensation including allodynia and hyperalgesia. Medical treatment typically includes antivirals, tricyclic antidepressants, anticonvulsants, corticosteroids, and opioid analgesics, but is often ineffective. ${ }^{1}$

So how could blockade of the sphenopalatine ganglion (SPG) improve symptoms of post herpetic ophthalmic neuralgia as seen in our case report? Although the mechanism by which the SPG block alleviates pain in headache disorders is not completely understood, it is likely multifactorial. The SPG is classified as a parasympathetic ganglion because it contains parasympathetic cell bodies. Preganglionic parasympathetic neurons synapse on cell bodies within the ganglion; however, post-ganglionic sympathetic neurons and somatic sensory afferent branches of the maxillary division of the trigeminal nerve also pass through the ganglion. ${ }^{2,3}$ The nerves leaving the SPG include the nasopalatine nerve, greater palatine nerve, lesser palatine nerve, posterior, superior and inferior lateral nasal branches, and pharyngeal branch of the maxillary nerve., ${ }^{2,4}$ There are also orbital branches, which pass directly to the apex of the orbit and eventually the lacrimal gland. ${ }^{2,5,6}$

The SPG has been implicated in multiple headache and pain syndromes. All of the above mentioned nerves can be affected by 
the SPG block. Currently accepted indications for the SPG block are sphenopalatine neuralgia, trigeminal neuralgia, atypical facial pain, acute migraine, acute and chronic cluster headaches, herpes zoster involving the ophthalmic nerve, and a variety of other facial neuralgias. ${ }^{2}$ In regards to post herpetic ophthalmic neuralgia, the direct connection to the maxillary branch of the trigeminal nerve may explain why blockade of the SPG is beneficial. Additionally, Ooman et al recently reported an undescribed branch of the SPG that likely contains sensory fibers and runs between the ophthalmic nerve and the SPG. This branch may explain additional anatomic findings for pain relief in the ophthalmic area after SPG blockage. ${ }^{7}$

Many techniques of the SPG block have been described. The SPG is located in the posterior wall of the nasopharynx just a few millimeters deep to the mucosa. Because of this superficial location, topical application of local anesthetic solution can diffuse across the nasal mucosa to block the SPG. We utilized the transnasal technique with a cotton tipped applicator. After the cotton tipped applicator was soaked in $4 \%$ lidocaine, it was then advanced in a horizontal plane along the middle turbinate using the zygomatic arch as a parallel reference structure, until it reached the posterior wall of the nasopharynx. It was then left in place for 20 minutes with repeated drops of $4 \%$ lidocaine every 3-4 minutes. The advantages of this transnasal approach are abundant, including the noninvasive nature without the need for needles, very low complication rates, technical ease, and repeatability. ${ }^{8}$

In this case report, we demonstrate the effectiveness of this technique in treating severe, debilitating post herpetic ophthalmic neuralgia and advocate for an increased consideration of the SPG block to provide pain relief for refractory post herpetic ophthalmic neuralgia.

\section{Acknowledgments}

None.

\section{Conflicts of interest}

The authors have no financial or conflicts of interest in this article.

\section{References}

1. Rae-Grant, Alexander, Zbys Fedorowicz, et al. Postherpetic Neuralgia. Dynamed Plus; 2017.

2. Windsor RE, Jahnke S. Sphenopalatine Ganglion Blockade: A Review and Proposed Modification of the Transnasal Technique. Pain Physician. 2004;7(2):283-286.

3. Waxman S. Correlative Neuroanatomy. 23rd ed. Appleton \& Lange: Stamford, USA; 1996. 265-266 p.

4. Gray H. The trigeminal nerve. In: Lewis WH, Anatomy of the Human Body. Lea \& Febiger: Philadelphia, USA; 1918. 886-899 p.

5. Ruskell GL. Distribution of pterygopalatine ganglion efferents to the lacrimal gland in man. Exp Eye Res. 2004;78(3):329-335.

6. Rusu MC, Pop F. The anatomy of the sympathetic pathway through the pterygopalatine fossa in humans. Ann Anat. 2010;192(1):17-22.

7. Oomen KP, Ebbeling M, de Ru JA, et al. A previously undescribed branch of the pterygopalatine ganglion. Am $J$ Rhinol Allergy. 2012;26(1):e40-45.

8. Piagkou M, Demesticha T, Troupis $\mathrm{T}$, et al. The Pterygopalatine Ganglion and its Role in Various Pain Syndromes: From Anatomy to Clinical Practice. Pain Practice. 2012;12(5):399-412. 